\title{
Change of Estrogenic Activity and Release of Chloride Ion in Chlorinated Bisphenol A after Exposure to Ultraviolet B
}

\author{
Yasuko Mutou, ${ }^{a, c}$ Yuko IBuki, ${ }^{*, a}$ Yoshiyasu Terao, ${ }^{b}$ Shuji KoJIma, ${ }^{c}$ and Rensuke $\mathrm{Goto}^{a}$ \\ ${ }^{a}$ Laboratory of Radiation Biology, Graduate School of Nutritional and Environmental Sciences, University of Shizuoka; \\ ${ }^{b}$ Laboratory of Environmental Chemistry and COE Program in the 21st Century, Graduate School of Nutritional and \\ Environmental Sciences, University of Shizuoka; 52-1 Yada, Shizuoka 422-8526, Japan: and ${ }^{c}$ Department of Radiation \\ Biosciences, Faculty of Pharmaceutical Sciences, Tokyo University of Science; Noda, Chiba 278-8510, Japan. \\ Received March 29, 2006; accepted July 10, 2006; published online July 13, 2006
}

\begin{abstract}
Bisphenol A (BPA) and chlorinated bisphenol A (CIBPAs) were detected in wastewater from waste paper recycling plants. In previous study, we showed the acute cytotoxicity of oxidized products of BPA and CIBPAs generated by ultraviolet (UV) irradiation. However, estrogenic activities of these photoproducts have not been studied. Therefore, we investigated change of estrogenic activities of BPA and CIBPAs [3-chlorobisphenol A (3CIBPA), 3,3'-dichlorobisphenol A (3,3'-diCIBPA) and 3,3',5-trichlorobisphenol A (3,3',5-triCIBPA)] after UVB irradiation using yeast two-hybrid assay. The agonist activities of CIBPAs were higher than that of BPA in the absence of S9. CIBPAs irradiated with UVB lost agonist activities. The addition of S9 also completely erased the activity. The antagonist activities of BPA and CIBPAs with or without UVB irradiation were not detected both in the absence or presence of S9. UVB irradiation $\left(0-100 \mathrm{~J} / \mathrm{cm}^{2}\right)$ decreased the agonist activity of 3,3 -diClBPA in proportion to increase of released chloride ion. The agonist activity was completely lost at $50 \mathrm{~J} / \mathrm{cm}^{2} \mathrm{of} \mathrm{UVB}$, of which dose could dissociated almost all chlorine. These findings suggested that UVB irradiation could decrease the estrogenic activity of chlorinated compounds, which was due to the selective release of chloride ion.
\end{abstract}

Key words chlorinated bisphenol A; ultraviolet B; bisphenol A; estrogen; photodegradation; endocrine disrupting compound

Bisphenol A (BPA) has been reported to cause modulating or disrupting the endocrine system in wildlife and human, ${ }^{1-3)}$ and to be detected in river water at more than $50 \%$ of locations investigated in Japan. ${ }^{4)}$ It is used as a material of polycarbonate plastic and epoxy resins, and as a developer in dyes for thermal papers. It was easily chlorinated at the bleaching process in waste paper recycling plants by sodium hypochlorite and then chlorinated BPAs (ClBPAs) were release into wastewater. ${ }^{5}$

Recently, several ClBPAs (3-chlorobisphenol A (3ClBPA), 3,3'-dichlorobisphenol A (3,3'-diClBPA) and 3,3',5-trichlorobisphenol A (3,3',5-triClBPA)) were described to interact more strongly with estrogen receptors than BPA. ${ }^{6,7)}$ The affinity of 3-CIBPA and 3,3'-diClBPA for human estrogen receptor was higher than that of BPA, however, the in vivo estrogenic activity of them in ovariectomized rats was comparable to that of BPA. ${ }^{8)}$ Furthermore, it was reported that ClBPAs inhibited 3,3',5-triiodothyronine (T3) binding to a plasma thyroid hormone-binding protein, transthyretin (TTR) of chicken and frog more strongly than BPA. ${ }^{4)}$ These results indicated that ClBPAs were endocrine disrupting compounds in the environmental and potent competitors of TTR.

Many chemical compounds in environment are degraded by bacteria metabolism and photooxidation. BPA is mainly metabolized through oxidative rearrangement by bacteria in the river water. $\left.{ }^{9}\right)$ Watanabe et al. described that BPA could be decomposed photocatalytically under ultraviolet (UV)-illumination in aqueous $\mathrm{TiO}_{2}$ despersion. ${ }^{10)}$ Zhou et al. indicated the photooxidation of BPA in water in the presence of ferric and oxalate ions under a $125 \mathrm{~W}$ high pressure mercury lamp. ${ }^{11)}$ Eriksson et al. showed that brominated and chlorinated BPA in aqueous were dehalogenated to isopropylphenol derivatives by UV irradiation. ${ }^{12)}$ We already reported the changes of acute cytotoxicity and chemical structure of BPA and ClBPAs after UV irradiation. However, the study of estrogenic activity of photoproducts is lacking. In this study, we investigated the change of estrogenic activities of BPA and CIBPAs after UV irradiation using yeast two-hybrid assay. The agonist and antagonist activities of BPA and ClBPAs exposed to UVB disappeared, which was related to release of chloride ion.

\section{MATERIALS AND METHODS}

Chemicals BPA $(>99 \%)$ was purchased from Kanto Chemical (Tokyo, Japan). ClBPAs (3-ClBPA, 3,3'-diClBPA, and 3,3',5-triClBPA) were synthesized according to previously described procedures. ${ }^{5)}$ All solvents used were of pesticide analysis grade.

$17 \beta$-Estradiol (E2) (>98\%), trans-stilbene (T-S) (95\%), and 4-hydroxytamoxifen (4H-TF) $(98 \%)$ were purchased from Sigma Chemical (St. Louis, MO, U.S.A.). Aurora ${ }^{\mathrm{TM}}$ Gal-XE Kit (Galacto-reaction buffer diluent, Light emission accelerator, and Glacton ${ }^{\mathrm{TM}}$ chemiluminescent substrate) was obtained from ICN Biomedicals (Aurora, OH, U.S.A.). Zymolyase 20T was a product of Seikagaku Kogyo (Tokyo, Japan). Rat liver S9 was purchased from Kikkoman (Japan). The yeast cells (Saccharomyces cervisiae Y190) with human estrogen receptor ER $\alpha$ and the coactivator TIF2 was kindly provided by Prof. Shinya Hashimoto (University of Shizuoka, Japan).

UVB Irradiation All chemicals were dissolved in ethanol and kept in the dark at $4{ }^{\circ} \mathrm{C}$ as $50 \mathrm{~mm}$ stock solutions. BPA and ClBPAs solutions $(1 \mathrm{ml})$ at $100 \mu \mathrm{M}$ were prepared by diluting the appropriate volumes of stock solutions with Milli-Q Water $(0.2 \%$ ethanol) in a glass dish $(19 \mathrm{~mm}$ diameter and $11 \mathrm{~mm}$ height). A UVB lamp (HP-30LM; Atto, Japan) with a $280-320 \mathrm{~nm}$ emission and a max. peak of $312 \mathrm{~nm}$ was used. The fluence was simultaneously measured 
and integrated using a radiometer (ATV-3W; Atto, Japan) with a $312 \mathrm{~nm}$ detector that was placed at the same distance as a glass dish from the UV source. The approximate irradiance of UVB at the sample level was $1.0 \mathrm{~mW} / \mathrm{cm}^{2}$. During irradiation, the dishes were covered with a polyethylene film. The weight of the glass dish was maintained by adding purified water every $2 \mathrm{~h}$ to prevent any decrease of water volume.

Estrogenic Activity by Yeast Two-Hybrid Assay. Measurement of Agonist Activity Chemicals were dissolved in dimethylsulfoxide (DMSO) and kept in the dark at $-20^{\circ} \mathrm{C}$ as stock solutions (E2; $200 \mathrm{~nm}$, T-S; $1 \mathrm{~mm}$, 4H-TF; $1 \mathrm{~mm}$, BPA; $1 \mathrm{~mm}, \mathrm{ClBPAs} ; 100 \mu \mathrm{M})$. The changes of estrogenic agonist activity of BPA and CIBPAs after UVB irradiation was measured using yeast two-hybrid assay described by Shiraishi et $a l{ }^{13)}$ E2 and T-S were used as positive controls for agonist assay in the absence and presence of S9, respectively. The agonist assay was performed with and without rat liver S9 to investigate an effect by metabolic activation. The test solutions $(20 \mu \mathrm{l})$ without S9 $(-\mathrm{S} 9)$ were diluted by $480 \mu \mathrm{l}$ of modified synthetic dropout medium lacking tryptophan and leucine (MSD med.). S9 mixture was prepared at concentration of $4.7 \%$ using $\mathrm{Z}$ buffer $\left(\mathrm{Na}_{2} \mathrm{HPO}_{4} ; 60 \mathrm{~mm}, \mathrm{Na}_{2} \mathrm{H}_{2} \mathrm{PO}_{4}\right.$; $40 \mathrm{~mm}, \mathrm{KCl} ; 10 \mathrm{~mm}, \mathrm{MgSO}_{4} ; 1 \mathrm{~mm}, \beta-\mathrm{NADP}^{+} ; 1.8 \mathrm{~mm}$, glucose-6-phosphate; $2.6 \mathrm{~mm}$, HEPES; $15 \mu \mathrm{M}$ ). Test solutions $(20 \mu \mathrm{l})$ containing $480 \mu \mathrm{l}$ of S9 mixture were treated at $37^{\circ} \mathrm{C}$ for $1 \mathrm{~h}$. The yeast cells were preincubated overnight at $30^{\circ} \mathrm{C}$ in MSD med., which were prepared to appropriate cell concentration (OD; $595 \mathrm{~nm}$ ) with MSD med. The yeast cells $(60 \mu \mathrm{l})$, test solution $(60 \mu \mathrm{l})$, and MSD med. $(60 \mu \mathrm{l})$ were incubated for $4 \mathrm{~h}$ at $30^{\circ} \mathrm{C}$ in a 96-well microplate. After incubation, the cells were digested with $80 \mu \mathrm{l}$ of lysis solution (zymolyase $20 \mathrm{~T} / \mathrm{Z}$ buffer $2 \mathrm{mg} / \mathrm{ml}+\mathrm{AURORA}$ reaction buffer sol.) at $37^{\circ} \mathrm{C}$ for $1 \mathrm{~h}$. AURORA reaction buffer sol. contained $1 \%$ Glacton $^{\mathrm{TM}}$ chemiluminescent substrate. The yeast cells produce $\beta$-galactosidase according to activity of estrogen. Chemiluminescent substrate enzymatically was cleaved by $\beta$-galactosidase. Chemiluminescent intensity (CLN) was measured using Luminoskan (Labsystems Research Centre, Finland). Agonist activity of a test compound was evaluated by $\mathrm{EC}_{\times 10}$ that was defined as the concentration at which the ratio of the chemiluminescent signal of the sample to that of the control is 10 . Relative value shows the ratio of an agonist activity of chlorinated BPA to that of BPA.

Measurement of Antagonist Activity The antagonist activity of BPA and CIBPAs after UVB irradiation was measured using yeast two-hybrid assay described by Shiraishi et $a l{ }^{13)} 4 \mathrm{H}-\mathrm{TF}$ as a positive control was dissolved in DMSO at concentration of $500 \mu \mathrm{M}(-\mathrm{S} 9)$ and $100 \mu \mathrm{M}(+\mathrm{S} 9)$. Test solutions $(20 \mu \mathrm{l})$ containing $480 \mu \mathrm{l}$ of $\mathrm{S} 9$ mixture were treated for $1 \mathrm{~h}$ at $37^{\circ} \mathrm{C}$. The yeast cells $(60 \mu \mathrm{l})$, test solution $(60 \mu \mathrm{l})$, and MSD med. containing $600 \mathrm{pm} \mathrm{E2}(60 \mu \mathrm{l})$ were incubated at $30^{\circ} \mathrm{C}$ for $4 \mathrm{~h}$. The values of antagonist activity represent the ratio of the inhibition (\%) of the CLN intensity of the test sample to that of a blank control.

Quantitative Determination of Chloride Ion Concentration Concentration of chloride ion released from chlorinated BPAs $(100 \mu \mathrm{M})$ after UVB irradiation was measured by a Ion Chromatography (IC) (Dionex, U.S.A.). All chromatographic analysis was performed using a DX-500 and a CD20 conductivity detector equipped with IonPac AS-12A anion separator column $(4 \times 250 \mathrm{~mm})$. The IC conditions were as follows: injection volume, $100 \mu \mathrm{l}$; eluent flow-rate, $1 \mathrm{ml} / \mathrm{min}$; eluent composition, $0.3 \mathrm{~mm} \mathrm{NaHCO}_{3} / 2.7 \mathrm{~mm} \mathrm{Na} \mathrm{CO}_{3}$. Milli $\mathrm{Q}$ water was used for all eluent, sample, and standard preparation. Chlorine standard solutions $(0-10 \mathrm{mg} / \mathrm{l})$ were adjusted using sodium chloride in Milli Q water.

\section{RESULTS AND DISCUSSION}

Estrogenic Activities of BPA and CIBPAs after UVB Irradiation. Agonist Activity The agonist activities of test compounds after UVB irradiation were measured by yeast two-hybrid assay. ${ }^{13)}$ Saccharomyces cerevisiae Y190 used in this assay has been introduced two expression plasmids, pGBT9-ERLBD and pGAAD424-TIF2, which carry a $\beta$ galactosidase reporter gene and require tryptophan and leucine for growth. ${ }^{1)}$ Agonist activity was represented by the ratio of the chemiluminescent intensity with $\beta$-galactosidase expression of the treated sample to that of a blank control.

The agonist activity of E2 without S9 increased dose-dependently at concentration ranges from 0 to $500 \mathrm{pm}$ and $\mathrm{EC}_{\times 10}$ was $180 \mathrm{pm}$. ClBPAs also indicated a dose response relationship in the range of concentration tested (Fig. 1A). The activity of BPA was not detected at concentration ranges from 0 to $1000 \mathrm{~nm}$. The $\mathrm{EC}_{\times 10}$ values and relative activities calculated are presented in Table 1. 3,3'-diClBPA showed the most powerful activity among all test compounds $\left(\mathrm{EC}_{\times 10}=44 \mathrm{~nm}\right)$ and the relative activity was 105 -fold higher than that of BPA. The activities of 3-ClBPA and 3,3',5-triClBPA were 12-fold and 35-fold, respectively. Furthermore, we examined agonist assay in the presence of S9. T-S used as a positive control showed a dose response relationship at concentration ranges from 0 to $2500 \mathrm{~nm}$ and $\mathrm{EC}_{\times 10}$ was $4200 \mathrm{~nm}$. BPA and ClBPAs showed no agonist activity in the presence of S9. All compounds might be metabolized by S9 and decreased their activities (Fig. 1B).

Nishihara et al. described that the most of the estrogen positive compounds have a phenol ring with a moiety of appropriate hydrophobicity at the para-position, and that substitution of a bulky moiety at the ortho-position reduces the activity. ${ }^{1)}$ However, in this study, ClBPAs without S9 showed stronger agonist activities than BPA, showing an enhancement of estrogenic agonist activity by chlorination. Hu et al. was also found that the estrogenic activity of the chlorinated aqueous BPA was 24 times that before chlorination. ${ }^{7)}$

ClBPAs completely lost their agonist activities by UVB irradiation $\left(100 \mathrm{~J} / \mathrm{cm}^{2}\right)$ in the presence or absence of $\mathrm{S} 9$ (Figs. $1 C, D)$. These results suggested that UVB irradiation degradated these compounds and/or produced photoproducts having no estrogenic activity. We have already reported that ClBPAs were changed to photoproducts such as 3-hydroxybisphenol A (3-OHBPA) by UVB irradiation $\left(100 \mathrm{~J} / \mathrm{cm}^{2}\right)$ and decomposed by higher UVB irradiation $\left(1000 \mathrm{~J} / \mathrm{cm}^{2}\right)$ in aqueous solution. ${ }^{14)}$ Since ClBPAs had absorption spectra of wavelengths ranging from 200 to $300 \mathrm{~nm}$ (data not shown), in almost agreement with that of UVB, these compounds were directly activated by UVB irradiation and then might be converted to other compounds, such as 3-OHBPA. ${ }^{14)}$

Antagonist Activity In antagonist assay, 4H-TF, a positive control, inhibited E2 receptor binding in a dose dependent manner at concentration ranges from 0 to $5000 \mathrm{~nm}(-\mathrm{S} 9)$ and from 0 to $500 \mathrm{~nm}(+\mathrm{S} 9)$, but their activities of BPA $(0$ 

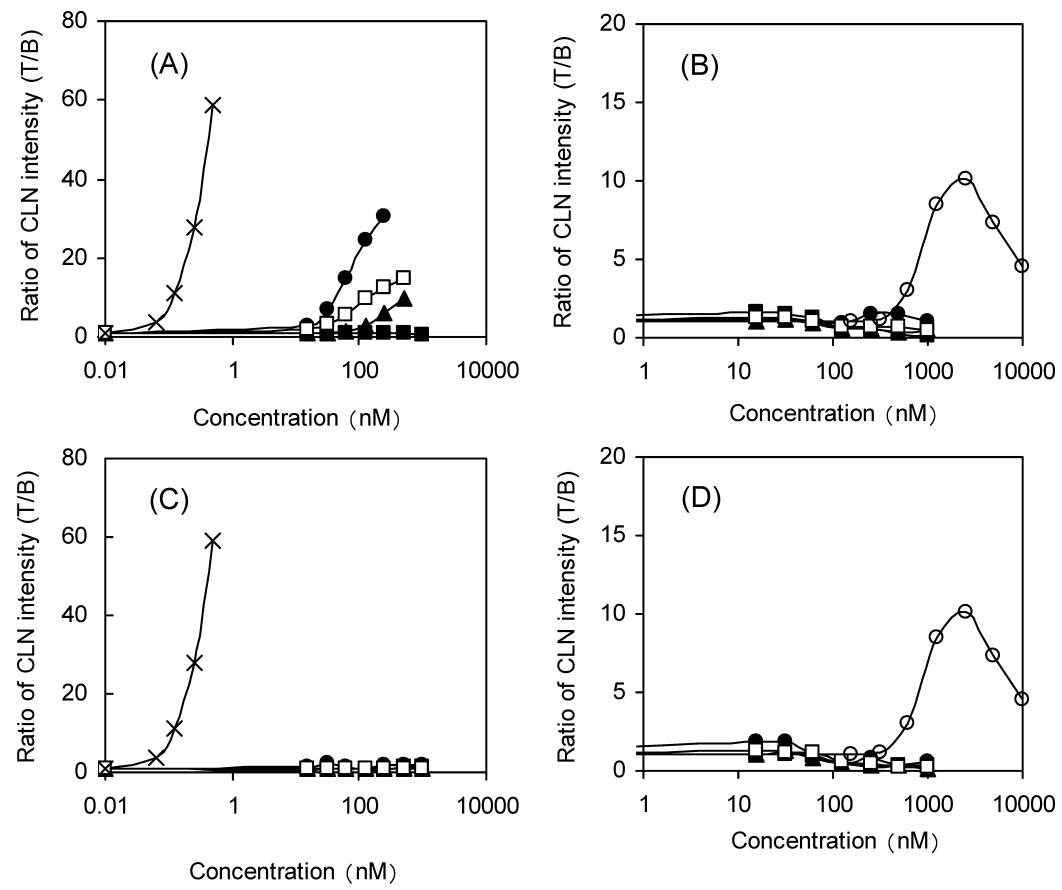

Fig. 1. Agonist Activity of Test Compounds Using Yeast Two-Hybrid Assay

The values of agonist activity represent the ratio (T/B) of the chemiluminescent (CLN) intensity of the treated sample (T) to that of a blank control (B). (A) Non-irradiation, without S9, (B) non-irradiation, with S9, (C) irradiation, without S9, (D) irradiation, with S9. $\mathbf{\square}$ : BPA, $\mathbf{\Delta}$ : 3-ClBPA, O: 3,3'-diClBPA, $\square:$ 3,3',5-triClBPA, X: E2, O: T-S.

Table 1. Estrogenic Activities of BPA and Chlorinated BPAs Using an Agonist Assay without S9 in the Yeast Two-Hybrid System

\begin{tabular}{lrc}
\hline \hline Compound & $\mathrm{EC}_{\times 10}(\mathrm{~nm})^{a)}$ & Relative value $^{b)}$ \\
\hline BPA & 4600 & 1 \\
3-ClBPA & 400 & 12 \\
3,3'-diClBPA & 44 & 105 \\
$3,3^{\prime}, 5$-triClBPA & 130 & 35 \\
\hline
\end{tabular}

a) $\mathrm{EC}_{\times 10}$ was defined as the concentration which the ratio of the chemiluminescent signal of the sample to that of a blank control was 10 . b) Relative value shows the ratio of an agonist activity of chlorinated BPA to that of BPA.

$1000 \mathrm{~nm})$ and ClBPAs (0-1000 nM) with or without S9 were not detected (data not shown). BPA and ClBPAs after UVB irradiation $\left(100 \mathrm{~J} / \mathrm{cm}^{2}\right)$ also showed no antagonist activity in the presence or absence of S9 (data not shown).

Chloride Ion Concentration and Agonist Activity of BPA and CIBPAs after UVB Irradiation To confirm participation of chlorine in estrogenic activity, we investigated the relationship the between the concentration of chloride ion released from 3,3'-diClBPA irradiated with UVB and agonist activity $(-\mathrm{S} 9)$. We selected $3,3^{\prime}$-diClBPA because it had the strongest cytotoxicity ${ }^{14)}$ and agonist activity as described in Fig. 1A.

Figure 2 showed the agonist activity of 3,3'-diClBPA $(100 \mu \mathrm{M})$ and concentration of chloride ion (\%) released after UVB irradiation $\left(0-100 \mathrm{~J} / \mathrm{cm}^{2}\right)$. Agonist activity has represented as a percentage of control (without irradiation). The concentration of whole chlorines in $3,3^{\prime}$-diClBPA is expressed as $100 \%$. Thirty three percent of the binding chlorine was released at UVB $5 \mathrm{~J} / \mathrm{cm}^{2}$, and the agonist activity decreased approximately half. Then, concentration of chloride ion released by UVB irradiation increased in a dose-dependent manner. Finally at $50 \mathrm{~J} / \mathrm{cm}^{2}$, the degree of dissociation reached $83 \%$, and the agonist activity was completely lost.

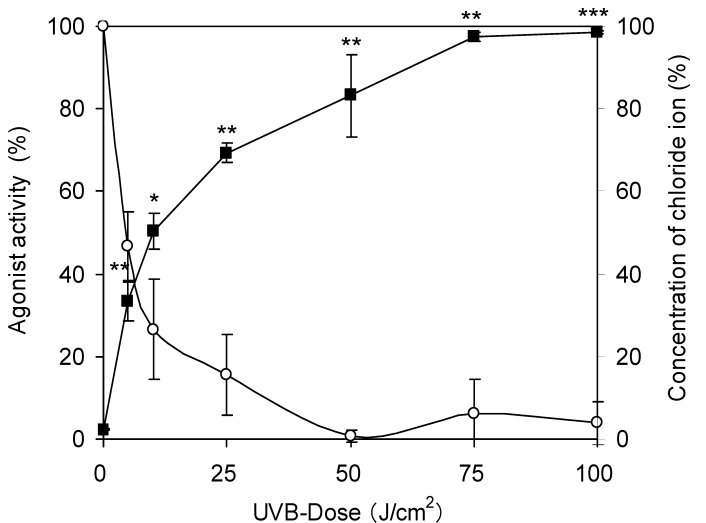

Fig. 2. Correlation between Agonist Activity and Concentration of Chloride Ion as a Function of UVB Dose

Agonist activity was represented as the percentage that $\mathrm{EC}_{\times 10}$ of nonirradiated sample was $100 \%$. The concentration of whole chlorines in 3,3'-diClBPA is expressed as $100 \%$. The results are expressed as means \pm S.D. $(n=3) . * p<0.001, * * p<0.0005$, $* * * p<0.0001\left(v s .0 \mathrm{~J} / \mathrm{cm}^{2}\right) .3,3^{\prime}$-diClBPA: $100 \mu \mathrm{M}$. Agonist activity $(\bigcirc)$, concentration of chloride ion ( $\mathbf{\square})$

UVB $5 \mathrm{~J} / \mathrm{cm}^{2}$ corresponded to the dose for more than several hours at noon on a fine summer day. This result suggested that sunlight exposure for a relative long time could lose estrogenic activity of chlorinated BPAs.

Recently, some important studies about photoreaction have been reported. Goto et al. showed the degradation of nonylphenol ethoxylates irradiated with $\mathrm{UVB}^{15)}$ and Hirahara et al. described similar phenomenon about the organophosphorous pesticides, fenthion and disulfoton. ${ }^{16)}$ Moreover, Hirahara et al. suggested that water was a critical factor in photolysis under UV-irradiation ${ }^{16)}$ and Kwok et al. showed that water played an catalysis role in the photodegradation. ${ }^{17)}$ In this study, since the exposure was carried out in water to investigate the photolysis under the environmental conditions, photoreaction of compounds might be promoted by water. 
In conclusion, we found that UVB irradiation decreased the estrogenic activities in BPA and ClBPAs, which was due to the dissociation of chlorine. The photodegradation was initiated at comparatively low doses of UVB. These results showed that UVB irradiation in sunlight was a very important factor to decompose of environmental pollutants. What influence many photoproducts produced by UV irradiation gives for ecosystem, is not yet definitely clarified. Further study of photolytic mechanism is necessary.

Acknowledgements We would like to express our profound gratitude to Prof. Shinya Hashimoto (University of Shizuoka, Japan) for his donation of the Saccharomyces cerevisiae Y190 used in this study. The authors are grateful to Dr. Yukinori Tani and Dr. Masaki Terasaki (University of Shizuoka, Japan) for their technical assistances.

\section{REFERENCES}

1) Nishihara T., Nishikawa J., Kanayama T., Dakeyama F., Saito K., Imagawa M., Takatori S., Kitagawa Y., Hori S., Utsumi H., J. Health Sci., 46, 282-298 (2000).

2) Paris F., Balaguer P., Terouanne B., Servant N., Lacoste C., Cravedi J. P., Nicolas J. C., Sultan C., Mol. Cell Endocrinol., 193, 43-49 (2002).

3) Krishnan A. V., Stathis P., Permuth S. F., Tokes L., Feldman D., Endocrinology, 132, 2279-2286 (1993).
4) Yamauchi K., Ishihara A., Fukazawa H., Terao Y., Toxicol. Appl. Pharmacol., 187, 110-117 (2003).

5) Fukazawa H., Hoshino K., Shiozawa T., Matsushita H., Terao Y., Chemosphere, 44, 973-979 (2001).

6) Fukazawa H., Watanabe M., Shiraishi F., Shiraishi H., Shiozawa T., Matsushita H., Terao Y., J. Health Sci., 48, 242-249 (2002)

7) Hu J. Y., Aizawa T., Ookubo S., Environ. Sci. Technol., 36, 19801987 (2002).

8) Takemura H., Ma J., Sayama K., Terao Y., Zhu B. T., Shimoi K., Toxicology, 207, 215-221 (2005).

9) Suzuki T., Nakagawa Y., Takano I., Yaguchi K., Yasuda K., Environ. Sci. Technol., 38, 2389 (2004).

10) Watanabe N., Horikoshi S., Kawabe H., Sugie Y., Zhao J., Hidaka H., Chemosphere, 52, 851-859 (2003).

11) Zhou D., Wu F., Deng N., Xiang W., Water Res., 38, 4107-4116 (2004).

12) Eriksson J., Rahm S., Green N., Bergman A., Jakobsson E., Chemosphere, 54, 117-126 (2004).

13) Shiraishi F., Shiraishi H., Nishikawa J., Nishihara T., Morita M., J. Environ. Chem., 10, 57-64 (2000).

14) Mutou Y., Ibuki Y., Terao Y., Kojima S., Goto R., Environ. Toxicol. Pharmacol., 21, 283-289 (2006).

15) Goto R., Kubota T., Ibuki Y., Kaji K., Goto A., Toxicology, 202, 237247 (2004)

16) Hirahara Y., Ueno H., Nakamuro K., J. Health Sci., 47, 129-135 (2001).

17) Kwok W. M., Zhao C., Li Y. L., Guan X., Wang D., Phillips D. L., J. Am. Chem. Soc., 126, 3119-3131 (2004). 